\title{
Bundle Visualization Strategies for HARDI Characteristics
}

\author{
Diana Röttger ${ }^{1}$, Daniela Dudai ${ }^{1},{\text { Dorit } \text { Merhof }^{2} \text {, and Stefan Müller }}^{1}$ \\ ${ }^{1}$ Institute for Computational Visualistics, University of Koblenz-Landau \\ ${ }^{2}$ Visual Computing, University of Konstanz
}

\begin{abstract}
In this paper we present visualization approaches for HARDI-based neuronal pathway representations using fiber encompassing hulls. We introduce novel bundle visualization techniques to indicate characteristics, such as information about tract integrity and multiple intra-voxel diffusion orientations. To accomplish this task, we developed an intra-bundle raycasting approach and use color mappings to encode diffusion characteristics on the bundle's surface. Additionally, we implemented a slicing approach using a plane orthogonal to the centerline of a bundle which reveals intra-bundle diffusion characteristics as well as the local bundle shape. With the presented approaches, we simultaneously reveal features of fiber bundles such as integrity or information about the underlying diffusion profile as well as context information, the shape of a current tract.
\end{abstract}

\section{Introduction}

Diffusion imaging is able to characterize organized tissue due to the fact that the movement of water molecules in fibrous material occurs in a larger scale with the fiber course than against it. It poses a large achievement in neuroscience, since it captures information about the organization of neuronal pathways in vivo. High angular resolution diffusion imaging (HARDI), was designed to overcome the limitations of diffusion tensor imaging (DTI) in terms of the ability to model complex intra-voxel diffusion profiles. The output is a probability density function on a sphere, the orientation distribution function (ODF), describing the movement of water molecules. Tractography techniques use the ODF to estimate trajectories and are best known representations of diffusion data. However, clinicians are oftentimes interested in the spatial position and shape of whole bundles: The generation of hulls approximating the reconstructed fibers can be beneficial in neurosurgical planning, for example, to determine the risk of an intervention [1]. Hulls are motivated by the fact that streamlines only approximate the diffusion process within a voxel; the hulls in turn approximate the reconstructed fibers. Conventionally, these hulls are single-colored and do not include information about the underlying diffusion profile. However, this information is essential in neuro-examinations and can be combined with hull visualization to improve tract-related examinations. Therefore, in this paper, we 
propose visual exploration approaches for HARDI combining both bundle morphology and intra-voxel fiber characteristics. Main contributions are: Raycastingbased visualizations of intra-bundle diffusion properties, using an evaluation of bundle characteristics on a ray from a vertex to the closest bundle centerline point. The information within a bundle is visualized on the geometry's surface using color maps. Centerline Slicing, a visual exploration method for diffusion characteristics using a plane, orthogonal to the centerline. The slicing reveals the bundle shape as well as diffusion characteristics for the current cross-section of the bundle. Visual enhancements including Phong illumination, ambient occlusion and silhouettes are integrated and can be activated by the user to facilitate depth perception. Therefore, the view does not suffer from visual cluttering and information about the global bundle orientation, shape, integrity as well as intra-voxel orientations is provided. The paper is organized as follows: In Section 2 related work on diffusion data visualization will be discussed. Section 3 will introduce the dataset and applied methods. Novel visualization approaches are proposed in Section 4 and results in Section 5 followed by a conclusion in Section 6.

\section{Related Work}

Tractography techniques aim to identify white matter tracts in the human brain and use either DTI or HARDI reconstructions [2-4]. These approaches provide essential information about the location and orientation of neuronal pathways, but conventionally do not include any indication about local tract properties such as integrity or fiber configuration. Therefore, a further visualization strategy for diffusion data exists, directly displaying the intra-voxel diffusion pattern using a geometry representation, known as glyphs. Kindlmann [5] presented a glyph representation for DTI, whereas Peeters et al. [6] introduced a fast rendering for HARDI. These approaches are beneficial in terms of analyzing local diffusion profiles precisely. However, the understanding of the global tract configuration, the course of the neuronal fibers, is lost. Therefore, visualizations based on line rendering but additionally including information of the local diffusion pattern arose. Zhang et al. [7] used Streamtubes and Streamsurfaces to visualize the local diffusion profiles of DTI. More precisely, the authors visualized linear diffusion tensor profiles using a tubular geometry whose orientation corresponds with the direction of the principal eigenvector of the diffusion tensor. In addition, the cross-section of a streamtube represents the ellipsis formed by the two remaining eigenvectors of the tensor at the position in the volume dataset. Klein et al. [8] presented an approach for DTI characteristic quantification on fiber bundles. The proposed method uses a resampled fiber bundle and computes an average principal fiber from the bundle. Afterwards, orthogonal planes are generated and used to compute fractional anisotropy (FA) values on the cross-section of the bundle. This approach is beneficial, since the quantification of DTI characteristics is possible for arbitrarily oriented fiber bundles and enables a tract-specific examination of diffusion properties. However, the 
simple approach presented for centerline reconstruction is not sufficient for complex bundle configurations.

A first step towards visualizing integrity information on bundle surfaces and slicing planes was made by Goldau et al. [9]. In their approach, the FA value is visualized along fiber bundles using either a color-coded slice or directly on the bundle boundary. For bundle color mapping, the authors compute a single FA value out of samples on a slice orthogonal to an average fiber's tangent. Subsequently they interpolate the obtained mean values of two neighboring planes to assign a characteristic to a specific vertex. This comprises many averaging steps and hence, the resulting index value is less representative in case of more complex fiber bundles. In our approach, we compute a ray for each individual vertex, resulting in a more precise representation of diffusion characteristics. In addition, we focus on HARDI fiber bundles and characteristics, compute a more accurate centerline and apply advanced visualizations.

\section{Data Acquisition and Precomputaion}

In the following, material and methods used for the presented approach will be introduced. Methods were implemented using MeVisLab, a development environment for medical image processing and visualization [10].

HARDI Dataset. The human brain dataset was of size $128 \times 128 \times 60$ and was acquired with a voxel size of $1.875 \times 1.875 \times 2 \mathrm{~mm}$ [11]. The applied gradient direction scheme included 200 directions and a b-value of $3000 \mathrm{~s} / \mathrm{mm}^{2}$.

Q-ball Imaging. Q-ball imaging, introduced by Tuch [12] is a method reconstructing the ODF from a HARDI signal and comprises a good balance in terms of acquisition requirements, computation time and a-priori assumptions. Descoteaux et al. [13] introduced an analytical solution using a spherical harmonics basis in combination with a Laplace-Beltrami regularization term. We evaluated the ODF using an icosahedron tessellation of order 3, resulting in 162 directions.

Tractrography. For HARDI-based fiber reconstruction, we used the approach proposed in Röttger et al. [14], a deterministic tractography approach based on the local ODF. The approach includes, amongst other aspects, the computation of distances to white matter boundaries using vectors orthogonal to the current orientation to determine the most adequate propagation.

Voxel Classification. Voxel classification was performed using a local ODFbased index for HARDI introduced by Röttger et al. [15]. It successfully categorizes a diffusion pattern into the following three compartments: isotropic diffusion profiles and anisotropic diffusion into single and multiple fiber populations. The index consists of a computation pipeline, in which at first a separation into white and gray matter takes place, followed by a classification into one and multiple fiber populations by analyzing the ODF. 
Hull Generation. The applied hull generation algorithm extends the approach proposed by Merhof et al. [1], consisting of the following four consecutive steps: volume rasterization, volume filtering, surface extraction and surface filtering. A binary fiber volume, the result of the rasterization stage, serves as the basis for isosurface computation. Our approach extends the proposed algorithm in terms of surface filtering: We include a reference volume (the rasterized fiber volume) which controls the adjustment of the geometric hull in the surface filtering stage.

Centerline Extraction. Several approaches of centerline reconstruction for fiber bundles exist in literature $[8,16]$. These approaches base on a principal fiber computation representing one centerline for a whole bundle. However, in cases of complex fiber configurations a principle fiber does not characterize a bundle appropriately, which is illustrated in Figure 1. Therefore, we applied a skeletonization approach (MeVisLab module DtfSkeletonization) using the fiber bundle morphology.

\section{Visualization Approaches}

Using the aforementioned precomputation results we will hereafter introduce the developed visualization techniques, which are illustrated in Figure 2. For enhanced three-dimensional understanding of the bundle shape, we apply a silhouette rendering approach, as well as Phong shading and ambient occlusion.

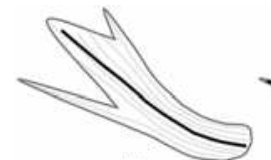

(a)

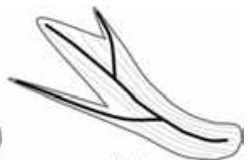

Fig. 1. Schematical illustration of a principle fiber (1a) and a skeleton (1b)

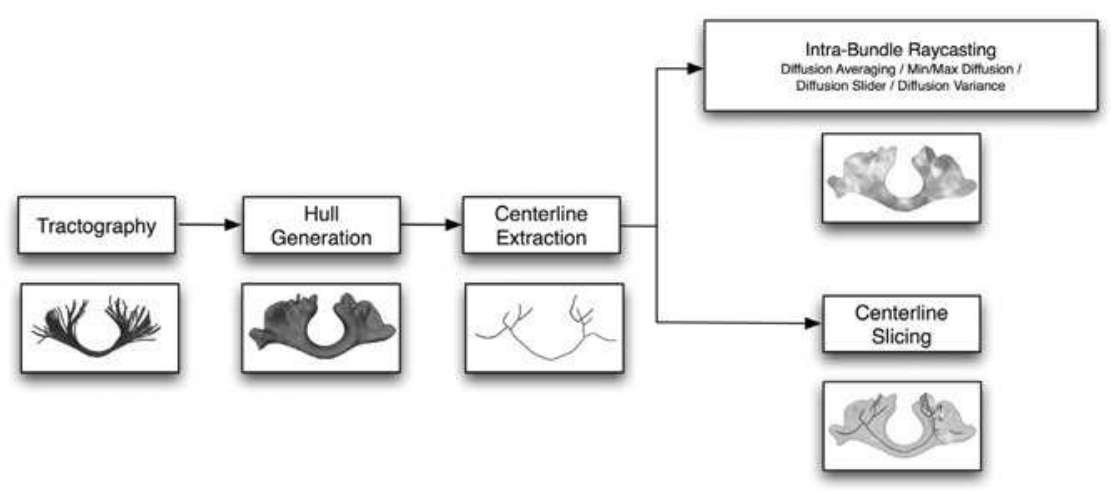

Fig. 2. Computation pipeline for HARDI-characteristic visualization. Preprocessing steps are shown in the left and intra-bundle visualizations in the right part. 


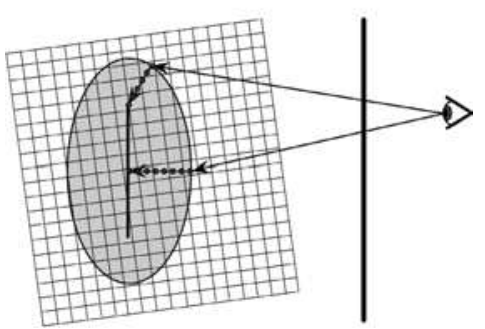

Fig. 3. Ray computation from the current vertex to the nearest centerline point

Silhouette rendering is performed by analyzing variances of neighboring normals in a deferred shading approach as introduced by Saito et al. [17]. Additionally, we implemented a Screen-Space Ambient Occlusion (SSAO) approach, first introduced by Mittring [18], which samples the depth buffer in the neighborhood of each fragment. The number of fragments closer to the viewport than the current one is used to darken the current fragment's color. However, combining color maps with enhanced rendering techniques to facilitate depth perception alters color appearance and can lead to false interpretations. Therefore, in our visualizations the user can interactively switch the rendering techniques on and off. In the following intra-bundle visualizations, we used two different color maps to emphasize the different meaning of both indices. For intra-bundle fiber configuration, red indicates single, yellow multiple and green isotropic diffusion. For variance encoding high variance is displayed in cyan and low variance in blue.

\subsection{Intra-bundle Raycasting}

The first method introduced in this paper uses a novel intra-bundle raycasting approach, which is realized though a GPU shader pipeline, using the hull geometry as an input. The centerline of the fiber tract is encoded in a $2 \mathrm{D}$ texture and transferred to the GPU as well as a 3D texture comprising the diffusion characteristics, in this case the voxel classification volume. The vertex shader computes the texture coordinates for characteristic evaluation, as well as the nearest point of the centerline. Further, a ray is traced from the current hull's vertex to the obtained centerline point, as illustrated in Figure 3. This ray is used for diffusion characteristic evaluation. We developed several visualization strategies for intra-bundle raycasting, motivated by volume rendering and explained in the following.

Diffusion Averaging. For Diffusion Averaging, we compute samples along the ray from the vertex to the nearest centerline point using a certain step length and resulting in $n$ distinct positions. These samples are used to evaluate the diffusion characteristics 3D texture and compute the average out of all obtained values. The final fragment color is determined through color mapping and thereby reflects the average diffusion value from a vertex to the centerline. 
Min/Max Diffusion. The Min/Max Diffusion mode is motivated by the maximum intensity projection (MIP) method, a transfer function, projecting the maximum value along a ray to the image plane. Transferring the idea to our approach results in computing the minimum and maximum characteristic value along the ray and the use of this value for color mapping. Within this visualization, a single characteristic value is displayed.

Diffusion Slider. With the Diffusion Slider, the user can interactively examine diffusion characteristics along the ray. In this case, no averaging of diffusion values takes place. Instead, the ray is normalized and sampled at discrete points. The resulting single diffusion values are visualized directly on the bundle's surface. Therefore, visualizations of diffusion characteristics from the hull to the bundle's centerline are feasible.

Diffusion Variance. Using the Diffusion Variance mode, diffusion homogeneities as well as inhomogeneities within the bundle, are highlighted. We therefore compute the variance of diffusion characteristics along the traced ray.

\subsection{Centerline Slicing}

In addition to previously presented approaches, we implemented a second intrabundle visualization, the Centerline Slicing. A plane orthogonal to the tangent of a user specified point on the centerline is generated and utilized for visualizing the color-coded index through texture mapping. The user selects a point through a mouse click on a rendering of the centerline. Afterwards, a shader pipeline uses the precomputed centerline rendered by use of the GL_LINES primitive as an input and generates the plane as the output. The coordinates of the user-defined point are transferred to the shaders via uniforms. The geometry shader is designed to generate a plane if the first coordinates of the current primitive are within an interval around the selected centerline point. The tangent of this centerline segment acts as the normal for the plane and is computed by subtracting both coordinates of the primitive. In bifurcations no distinct centerline tangent exists, which can be used to generate the plane. Therefore, in this case we use the view-vector as the plane normal. Thus, the user can interactively examine the diffusion pattern while moving the camera and the plane origin remains unchanged. To enhance the spatial understanding of the bundle, we render the parts of the plane not lying within the bundle transparent. Therefore, the plane not only exhibits information about the diffusivity, but also reveals the shape of the cross-section of the bundle for arbitrarily oriented centerlines.

\section{Results and Discussion}

Since the presented approaches are designed for challenging pathway configurations, we focus on fibers running in the centrum semiovale, a region in the brain, where the following three fiber tracts meet: the corpus callosum, the corticospinal 


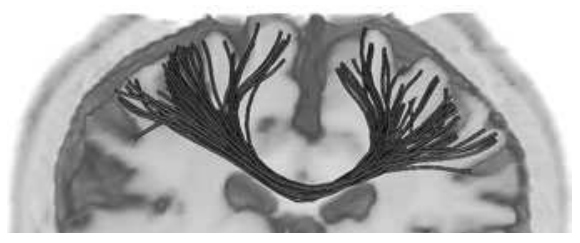

Fig. 4. Streamtube visualization of callosal fibers in the centrum semiovale

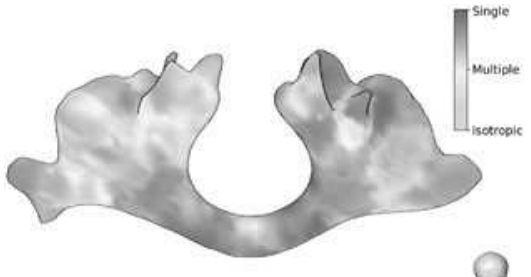

(a)

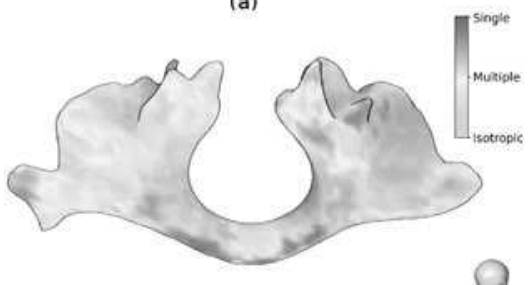

(c)

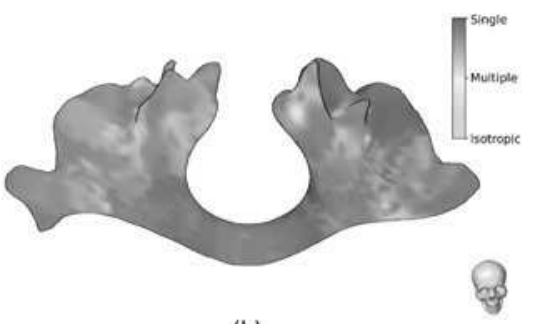

(b)

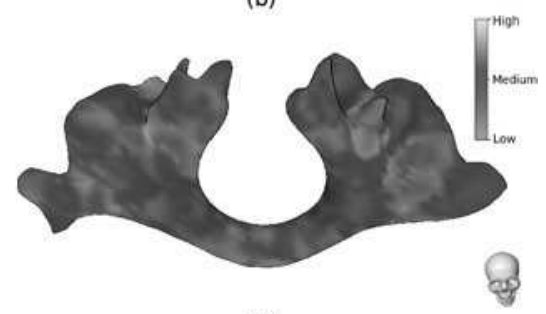

(d)

Fig. 5. Color mapping of values along the ray: Diffusion Averaging (5a) and Max Diffusion (5b) reveal multiple diffusion in the centrum semiovale, associated bundle parts appear yellow. Contrary, regions with highest integrity appear red using the Min Diffusion approach (5c). The Diffusion Variance highlights high diffusion variances (5d).

tract and the superior longitudinal fasciculus. Figure 4 shows fibers belonging to the corpus callosum, visualized as streamtubes. Results of the Diffusion Averaging, Max Diffusion, Min Diffusion and Diffusion Variance visualization on the hull without anatomical volume rendering are shown in Figure 5 and the Diffusion Slider, the Centerline Slicing as well as visual enhancements in Figure 6. Performance evaluation was accomplished on a Core2 Duo, $3.16 \mathrm{GHz}$ with 4 GB RAM and a NVIDIA GeForce GTX 285 graphics card. Following average frames per second (fps) were achieved for callosal fibers shown in this paper. The raycasting-based approach features $11 \mathrm{fps}$ when performing a $360^{\circ}$ rotation of the bundle. Ambient occlusion and silhouette visualization reduce the average fps to 6 . The Centerline Slicing mode achieved 22 fps on average.

Using the presented visualization approaches arbitrary neuronal pathway morphologies can be visualized with significant diffusion encoding color values. 


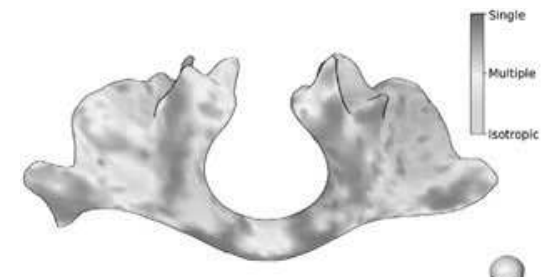

(a)

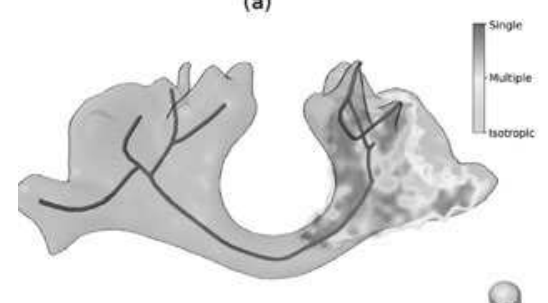

(c)

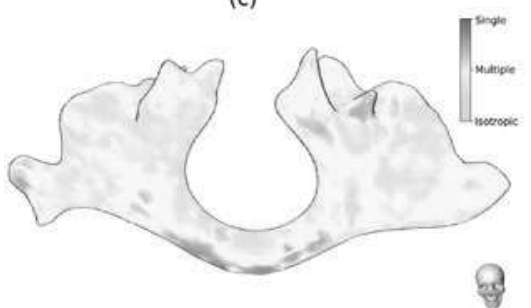

(e)

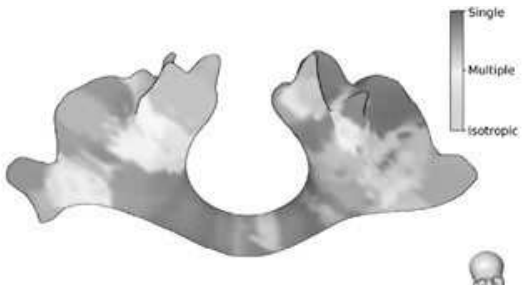

(b)

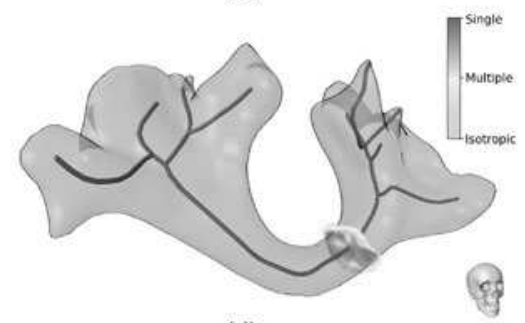

(d)

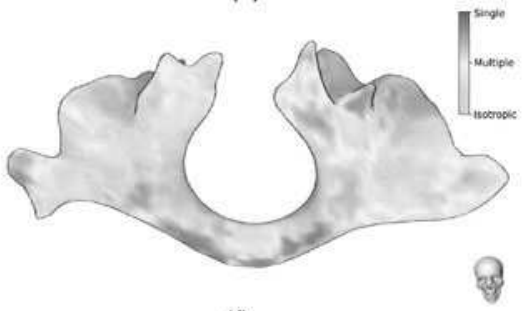

(f)

Fig. 6. Color mapping of the value on the hull (6a) and the centerline (6b) using the Diffusion Slider. Parts of the hull leaking into gray matter appear in green (isotropic diffusion). High single fiber configuration is obtained in the center of the corpus callosum. Centerline Slicing within regions of complex diffusion profiles, plane is oriented with the view-vector (6c) and in the center of the corpus callosum, plane is orthogonal to the centerline's tangent (6d). Phong illumination (6e) and ambient occlusion (6f) with applied silhouette rendering.

However, our method depends on the bundle's centerline. For complex morphologies, a detailed skeleton is crucial to result in reliable diffusion information, since the ray is determined by the closest centerline point. However, a detailed centerline reduces performance, since more points have to be considered for raycasting. In addition, the amount of diffusion information which is mapped through the ray to a bundle vertex, depends on the centerline's curvature and therefore is not uniformly distributed. In terms of large bundles or a less representative centerline the Diffusion Averaging visualization might not be representative, since all values on the ray are taken into consideration. This accounts for inhomogeneous bundles as well, since if the variance of diffusion values along the ray is too high a mean value can lead to false interpretation. In these cases a preliminary 
examination of the bundle using the Diffusion Variance or the Diffusion Slider mode and a subsequent adjustment of the centerline enhances the visualization.

Results were additionally discussed with seven neuroscientists to answer the following questions: Whether the approaches provide a better understanding as well as insight into the data, if the introduced perception enhancements facilitate depth impression as well as in which questions the approaches can pose a contribution. Findings resulting from the visualizations correspond with medical knowledge: The center of the corpus callosum comprises highest integrity as well as directionality. However, the region of the centrum semiovale features more complex fiber distributions. The Diffusion Averaging approach was one of the favorite visualization within experts: They stated it provides a good first impression about the underlying diffusion and that it is a straightforward visualization. Diffusion evaluation using the Diffusion Slider mode is of special interest, since the bundle skeleton can be considered to represent the whole bundle and visualizations are helpful in tract-based examinations. Furthermore, the Diffusion Variance visualization reveals interesting intra-bundle conditions: Regarding the center of the corpus callosum, we can identify similar values in both of the Min/Max Diffusion visualizations. These regions appear in purple and indicate low variance. Vice versa scenarios can be detected as well. The Centerline Slicing mode provides a more detailed examination, which can be beneficial in regions with high variance and therefore can be performed in a subsequent step. Considering the presented visual enhancements, all participants agreed that the combination of ambient occlusion and silhouettes as displayed in Figure 6 is the best depth encoding visualization. Color changes caused by Phong illumination are rated to be more violating than those caused by ambient occlusion. In general, experts think that the provided visualizations are of great interest and can be beneficial in questions related to neurosurgical planning, as well as disorder monitoring, for example in stroke patients.

\section{Conclusion and Future Work}

In this paper we proposed visualization techniques for intra-bundle diffusion characteristics using HARDI-based fiber reconstruction and diffusion profiles. Today, it is possible to describe intra-voxel diffusion patterns using HARDI classifiers. However an user-friendly and problem-specific visualization had been missing, although, a visual diffusion characteristics exploration in combination with tract morphology has great potential in many clinical questions. With our intra-bundle visualization approach we are taking a step forward towards a combination of global tract morphology and diffusion characteristics for HARDI. Considering HARDI fiber tracking results, an approach managing overlapping bundle regions is needed. Potential approaches include the use of focus and context rendering techniques. From the medical point of view it is interesting to evaluate the application of the presented visualizations to neurosurgical examinations. 


\section{References}

1. Merhof, D., Meister, M., Bingöl, E., Nimsky, C., Greiner, G.: Isosurface-based generation of hulls encompassing neuronal pathways. Stereotactic and Functional Neurosurgery, 50-60 (2009)

2. Basser, P.J., Pajevic, S., Pierpaoli, C., Duda, J., Aldroubi, A.: In vivo fiber tractography using DT-MRI data. Magnetic Resonance in Medicine 44, 625-632 (2000)

3. Lazar, M., Weinstein, D.M., Tsuruda, J.S., Hasan, K.M., Arfanakis, K., Meyerand, M.E., Badie, B., Rowley, H.A., Haughton, V., Field, A., Alexander, A.L.: White matter tractography using diffusion tensor deflection. Human Brain Mapping 18 (2003)

4. Descoteaux, M., Deriche, R., Knösche, T.R., Anwander, A.: Deterministic and probabilistic tractography based on complex fibre orientation distributions. IEEE Transactions on Medical Imaging 28 (2009)

5. Kindlmann, G.L.: Superquadric tensor glyphs. In: VisSym., pp. 147-154 (2004)

6. Peeters, T.H.J.M., Prckovska, V., van Almsick, M., Vilanova, A., ter Haar Romeny, B.M.: Fast and sleek glyph rendering for interactive HARDI data exploration. In: PacificVis., pp. 153-160 (2009)

7. Zhang, S., Demiralp, Ç., Laidlaw, D.H.: Visualizing diffusion tensor MR images using streamtubes and streamsurfaces. IEEE Transactions on Visualization and Computer Graphics 9, 454-462 (2003)

8. Klein, J., Hermann, S., Konrad, O., Hahn, H.K., Peitgen, H.O.: Automatic quantification of DTI parameters along fiber bundles. In: Bildverarbeitung für die Medizin (2007)

9. Goldau, M., Wiebel, A., Hlawitschka, M., Scheuermann, G., Tittgemeyer, M.: Visualizing DTI parameters on boundary surfaces of white matter fiber bundles. In: Proceedings of the Twelfth IASTED International Conference on Computer Graphics and Imaging, pp. 53-61 (2011)

10. MeVis Medical Solutions AG: MeVisLab, medical image processing and visualization (December 2011), http://www.mevislab.de

11. Poupon, C., Poupon, F., Allirol, L., Mangin, J.F.: A database dedicated to anatomo-functional study of human brain connectivity. In: 12th HBM Neuroimage, Florence, Italie, Number 646 (2006)

12. Tuch, D.S.: Q-ball imaging. Magnetic Resonance in Medicine 52, 1358-1372 (2004)

13. Descoteaux, M., Angelino, E., Fitzgibbons, S., Deriche, R.: Regularized, fast and robust analytical Q-ball imaging. Magnetic Resonance in Medicine 58, 497-510 (2007)

14. Röttger, D., Seib, V., Müller, S.: Distance-based tractography in high angular resolution diffusion MRI. The Visual Computer 27, 729-738 (2011)

15. Röttger, D., Dudai, D., Merhof, D., Müller, S.: ISMI: A classification index for high angular resolution diffusion imaging. In: Proceedings of SPIE Medical Imaging (2012)

16. Chen, W., Zhang, S., Correia, S., Ebert, D.S.: Abstractive representation and exploration of hierarchically clustered diffusion tensor fiber tracts. Computer Graphics Forum 27, 1071-1078 (2008)

17. Saito, T., Takahashi, T.: Comprehensible rendering of 3-D shapes. In: Proceedings of the 17th Annual Conference on Computer Graphics and Interactive Techniques, SIGGRAPH 1990, pp. 197-206. ACM, New York (1990)

18. Mittring, M.: Finding Next Gen - CryEngine 2. In: ACM SIGGRAPH 2007 Courses. SIGGRAPH 2007, pp. 97-121 (2007) 